\title{
URIDINE DIPHOSPHATE GLUCOSE: THE COENZYME OF THE GALACTOSE-GLUCOSE PHOSPHATE ISOMERIZATION
}

A PREvious report ${ }^{1}$ dealt with the enzymatic conversion of galactose-1-phosphate into glucose-1-phosphate and mentioned that a thermostable factor is necessary for the reaction. This factor has now been obtained practically pure, and for reasons given below will be referred to as uridine diphosphate glucosc.

The preparation of uridine diphosphate was effected by extraction of bakers' yeast with one volume of ethanol, followed by fractional precipitation with mercuric acetate, adsorption on charcoal, elution with ethanol and treatment with a cation-exchange resin.

During the last stages of purification, it was observed that the activity ran parallel with a substance showing absorption at 260 $\mathrm{m} \mu$. The same parallelism was observed during paper chromatography with 77 per cent ethanol. The absorption spectrum of the substance is identical with that of uridine, and shows the same changes in acid and alkali, and after treatment with bromine. For each uridine calculated. from its absorption coefficient ${ }^{2}$, the substance contains two phosphates and two nitrogens.

On mild acid hydrolysis, uridine diphosphate loses its coenzymatic activity and yields a molecule of a reducing sugar which was identified as glucose by selective fermentation, paper chromatography, and the carbazole reaction ${ }^{3}$ after removing interfering substances with ion exchange resins. The rate of glucose liberation in $0.01 \mathrm{~N}$ acid at $100 \% \mathrm{C}$. is higher than that of glucose-1-phosphate (halflife-time $\mathrm{t}_{1 / \mathrm{s}}=0.8$ and $3.8 \mathrm{~min}$. respectively).

Intact uridine diphosphate is non-reducing, and, tugether with the liberation of glucose, there occurs a change in the titration curve: it has two primary phosphoric acid groups and no secondary, while removal of the glucose unmasks a secondary acid group. This indicates that the glucose must be combined at position 1 with a phosphate group. Further evidence on the linkage of glucose to phosphate was obtained by treatment of uridine diphosphate with a suspension of washed kidney particles. A substance was liberated which behaved like glucose-1-phosphate under the action of phosphoglucomutase. Oxidation of uridine diphosphate with periodate gave one molecule of formic acid and no formaldehyde, as would be expected from a glucopyranosyl residue.

The substance remaining after removal of the glucose contains two phosphate groups, one of which is 90 per cent hydrolysed. in $N$ acid at $100^{\circ} \mathrm{C}$. in $15 \mathrm{~min}$. After hydrolysing this labile phosphate, the reaction mixture shows two primary and two secondary acid groups. The second phosphate group is very stable, and its rate of hydrolysis was found to be nearly equal to that given by Gulland and Smith ${ }^{4}$ for uridine-2-phosphate. However, uridine-5-phosphate cannot as yet be excluded.

The substance remaining after removal of the two phosphate groups was identified as uridine by paper chromatography. The pentose could be estimated by the orcinol reaction after bromination 5 . Further hydrolysis in $6 \mathrm{~N}$ hydrochloric acid for $2 \mathrm{hr}$. at $120^{\circ} \mathrm{C}$. gave uracil as judged by chromatography and by its spectrum at various $p \mathrm{H}$.

On the basis of this evidence, uridine diphosphate can be tentatively assigned the accompanying formula, which shows a pyrophosphate linkage and explains the facts that it has only two primary acid groups, and that one secondany acid group appears on removal of the glucose residue and another on hydrolysis of the labile phosphate. Molecular weight calculated from the dry weight and uridine content gave a value of 630 , which is not too far from the theoretical value of 566 .

One point is not yet clear; that is the action of alkali. Treatment with $0.1 \mathrm{~N}$ alkali at 
100 ' C. destroys the activity in a few minutes. No phosphate is liberated; but there occurs a stabilization of the glucosidic linkage so that the glucose now undergoes hydrolysis at the same rate as the first phosphate group. Presumably alkali brings about a rearrangement of the molecule.

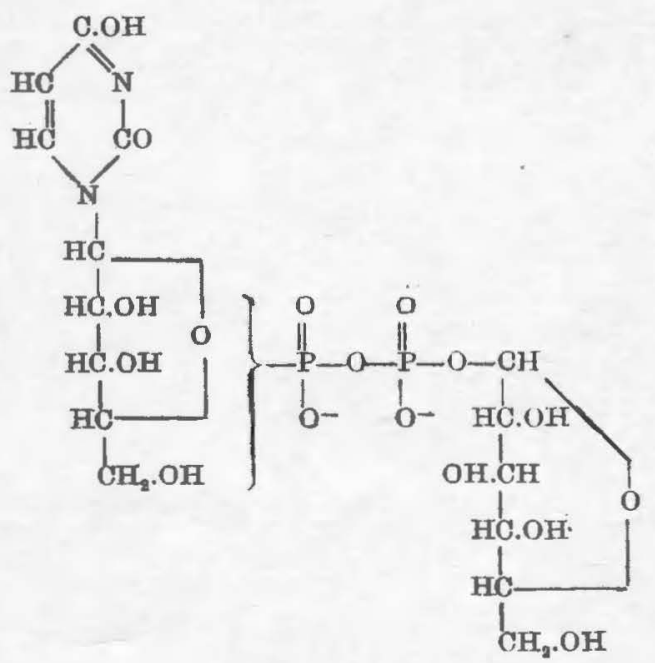

1. Caputto, R., leloir, L. F., Trucco, R. E. CarDini, C. E, and Paladini, A. C., J. Biol. Chem., 179, 497 (1949).

2. Plofser, J. MCT., and Lozing, H. S., Biol., Ghem., 178, $431(1949)$.

3. Gurix, S., and Hood, D. B., J. Biol. Chem., 131, 211 (1939).
The mechanism by which uridine diphosphate accelerates the conversion of galactose into glucose will require further investigation. The fact that glucose phosphate can be recognized as a part of the coenzyme suggests that this portion may exchange with the substrate during the reaction cycle.

An interesting fact is the similarity of uridine diphosphate with the compound which Park and Johnson ${ }^{6}$ have found to accumulate in Staphylococcus aureus grown in the presence of penicillin. It is not possible from their data to conclude that it is the same compound; but the resemblance is striking.

A full account of this investigation will be published elsewere.
C. E. Cardini
A. C. Paladini
R. Caputto
L. F. LELOIR

Instituto de Investigaciones Bioquímicas, Fundación Campomar, Julián Alvarez 1719, Buenos Aires.
4. Gulland, J. M., and Smrth, H., J. Chem. Soc., 338 (1947).

5. Massart, L., and Hoste, J., Biochim. et Biophys. Acta, 1, 83 (1947).

6. PARK, J. T., and Jorinson, M. J., J. Biol. Chem., 179, 585 (1949). 